\title{
Research Review of China Emergency Evacuation
}

\author{
$\mathrm{Hu} \mathrm{Yi}$ \\ China Research Center for Emergency Management \\ Wuhan University of Technology \\ Wuhan, P.R.China, 430070 \\ E-mail: whut-huyi@qq.com)
}

\begin{abstract}
The behavior of people in emergency evacuation is the hotspot in the field of security. This article introduces the current situation and development trend of emergency evacuation, reviews the behavior characteristics of evacuation, the length of evacuation time and scene experiment and constructs the evacuation model in the background of emergencies. At present, the research emphasis of China studies are still focusing on simulation, using computer to build emergent environment, studying the emergency evacuation behavior of people under fixed scene, adopting the more intelligent algorithm and evacuation model to enhance the authenticity of evacuation simulation. However, there are few studies on individual behavioral characteristics and group behavior in evacuation process. At the same time, the optimization of the model and the application of the model can be used as an important direction for future research.
\end{abstract}

Keywords-Emergencies; Simulation; Evacuation Model; Research prospect

\section{INTRODUCTION}

Public safety has attracted more and more attention with the development of social economy and expansion of urban scale since the "13th Five-Year Plan". Dense Population is an important feature of public places, which has seriously affected the security of public places. As Shown in Fig. 1, evacuation problem is becoming one of the most important security factors, more and more attention has been paid to it, and the evacuation behavior has become the research hotspot in the security field.

Under the emergency situation, studying the behavior characteristics and response laws of the crowd, conducting pertinent safe evacuation research, improving the emergency disposal ability, preventing and reducing the occurrence of emergency events, developing effective evacuation management strategy are of great practical significance to promote the construction of public emergency management and the harmonious development of society. At present, the research on the emergency evacuation behavior is mainly carried out from the aspects of evacuation behavior and evacuation time, scene experiment and evacuation model.

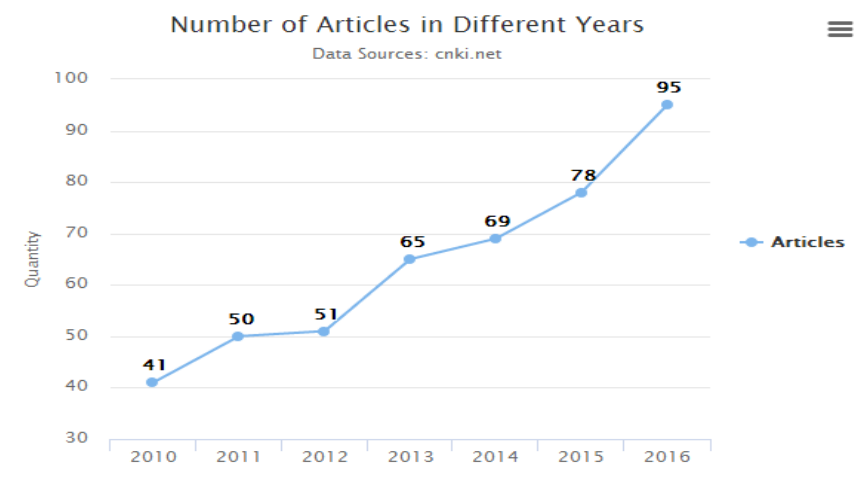

Fig. 1. Overview of Publications per Year

\section{BEHAVIORAL ChARACTERISTICS AND EVACUATION TIME}

The basic theory of behavioral characteristics in evacuation indicates that the behavioral characteristics and influencing factors of individuals themselves, individuals and groups at different stages are very different, after the occurrence of incidents or emergency situations, people will judge the rapidly changing environment based on available information and take different actions at different stages of the emergency situation. The scene of emergencies, the choice of evacuation paths, the understanding of safety knowledge, and the evacuation behavior as well as psychological characteristics can also affect the evacuation of people. The study of behavioral characteristics is mainly through the post hoc investigation of survivor or the analysis of videos, also can be obtained through the scene experiment. Xu Min [1], concluded that the main psychological factors influencing the behavioral characteristics in emergencies were panic and fear, and the coping styles of people of different ages were different in different places based on a large number of post-disaster reports. Zhan Xin [2], analysis the psychological behavior of evacuation through the questionnaire survey before and after the scene experiment, pointed out there were differences in evacuation behavior and the choice of evacuation routes of different age, sex and staff.

The time required for emergency evacuation is affected by factors such as the state of the emergency, reaction, decision, and action. Graat Ethel [3] suggests that the total evacuation time can be divided into three parts: the time of the perceived danger, the time taken to make the evacuation decision, and the time to move to the safe area as shown in Fig. 2. Tian Yumin[4] studied the distribution of evacuation delay time and its influence on evacuation time, proposed that the evacuation 
time mainly includes the delay time before evacuation and the evacuation time on passageway.

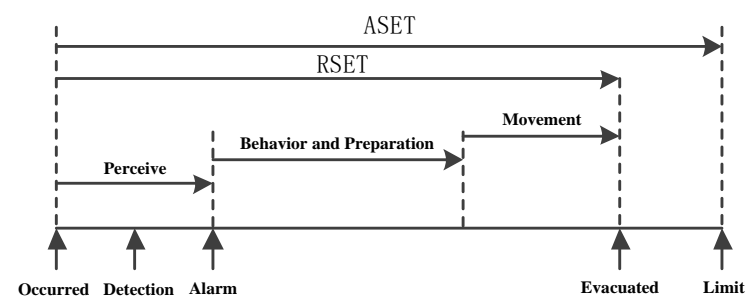

Fig. 2. Evacuation Time

\section{SCENE EXPERIMENT}

The general scene experiment is building a grid, step-by-step evacuation scene for evacuation, aiming at verifying the model, finding out the law of evacuation, in order to guide the actual. Through the layout of the scene forming different scene such as narrow channel, bottleneck through, the intersection of people and guidance information, requiring small groups bundled, to observe the result of evacuation and export choice of the crowd in an emergency situation, comprehensively weighing the distance of export and the difficulty of evacuation, the degree of acceptance of information and the influence of kinship and herd behavior. Huo FeiZhou [5], explored the effects of different evacuation patterns on step by step evacuation and overall evacuation through the scene experiment of high-rise building evacuation. At the same time some scholars build interaction, real-time, virtual environment through the actual three-dimensional visual and audio, instead of being exposed to the real extreme risk, people can use a safe way to simulate the disaster atmosphere in this way.

The study of escape behavior under virtual emergency environment and comparing with the actual investigation data achieved good results. Because of the complex characteristics of emergency evacuation, people under emergency evacuation are different from the general research object, it is hard to learn the influencing factors of evacuation through the scene experiments, the individual behavior is not only affected by the external factors, but also by the psychological status, in the conventional scene experiment, the interaction between the emergency scene and the personnel is low, so it is difficult to restore the law of crowd evacuation. Organizing scale of the scene experiment needs huge cost, facing a variety of unexpected risks in human, material, financial and operational, therefore, the computer simulation is still the main research method in the research of the evacuation problem. The scene experiment is usually used as an assistant means to collect the basic data of the evacuation and verify the reliability of evacuation simulation.

\section{EVACUATION MODEL}

The queuing model is the representative macro evacuation simulation model which considering the population as a whole with the development of computer technology, in late 1980s. Without considering the different individual's psychological reflection, personality characteristics and other individual behavior factors are gradually eliminated due to the weak application of simulation results. The microscopic model has become the hotspot of research based on the individual characteristics of the personnel. Nowadays it is a common method to use the computer simulation technology and even the virtual reality technology to study the evacuation problem in different scenes. In the virtual environment, studying evacuation need to consider a variety of factors, such as scene model, disaster model, personnel movement model, character psychological activities, human perception function and so on. Many scholars have done a lot of researches from different perspectives. These studies mainly focus on how to simulate the movement characteristics of people in the scene. In order to get a better understanding of evacuation models, we summarize the features of main evacuation models in Table I.

TABLE I. MODEL INTRODUCTION

\begin{tabular}{|c|c|c|}
\hline Model & Description & Features \\
\hline network model & $\begin{array}{l}\text { Network models represent a pedestrian facility as a graph } \mathrm{G}(\mathrm{N}, \mathrm{A}) \text {, where the set } \mathrm{N} \text { of } \\
\text { nodes represents the different rooms and the set A of arcs the links between them. }\end{array}$ & $\begin{array}{l}\text { Discrete } \\
\text { Simple }\end{array}$ \\
\hline cellular automata & $\begin{array}{c}\text { Cellular automata models are microscopic simulation models where pedestrians are } \\
\text { considered individually. They present the building lay-out by a grid divided into cells. } \\
\text { Usually, each cell can be occupied by a single pedestrian. }\end{array}$ & $\begin{array}{l}\text { Discrete Simple } \\
\text { Flexible } \\
\text { Non-linear }\end{array}$ \\
\hline
\end{tabular}

The agent-based model takes a bottom-up approach, in which only the behavior of multi-agent individual pedestrians is modeled, and the resulting interactions determine the macroscopic behavior. Each agent can have a unique set of behavioral rules that allow to model

Discrete Multi-level Diversified heterogeneity in the population
In this type of model, pedestrians have a desired velocity in the direction of their destination and their acceleration (deceleration) is the result of different forces. An individual experiences an attractive force in the direction of his target destination, and repulsive forces experiences an attractive force in the direction of his target destination,
from obstacles (e.g. walls) and other pedestrians.
Continuity Scalability Robustness social force
We counted the use of models in recent years in Fig. 3. Based on the multi agent model, Zhang Xuefeng [6], established the disaster mathematical model and the individual perception model, which combined the personnel simulation and disaster model, and showed the evacuation in the multi disaster environment. Liu Quanping [7], in order to reflect the 
group's integrity and individual differences, putting forward a kind of multi-agent and cellular automaton evacuation model, which better reflecting the individual personality, physical and psychological (panic degree) impact on evacuation behavior. Dong Chongjie [8], improved the cuckoo algorithm to solve the multi-objective optimization evacuation network model, achieving a good results. Wang Xia [9], took the degree of panic mentality into the social force model, considered the influence of herd behavior on the choice of direction, realistically reflecting the evacuation behavior of people in emergencies, providing a theoretical basis to guiding the emergency evacuation.

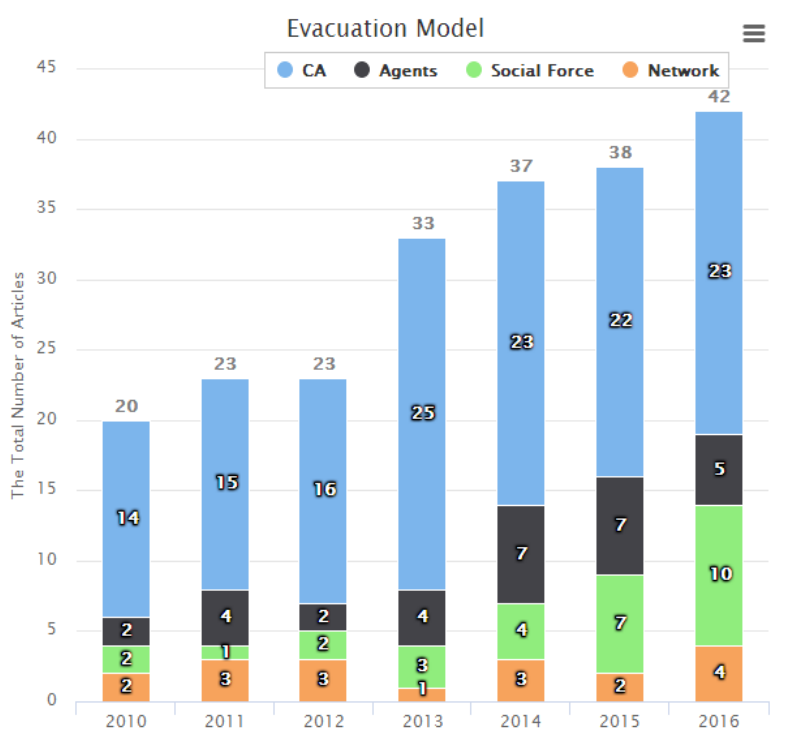

Fig. 3. Overview of Publications per Model and per Year

\section{Model Optimization AND APPLICATION}

Micro discrete model based on agent has become a research trend in recent years, focusing on the introduction of different models and algorithms to optimize the model, studying individual behavior and group effects. The model optimization generally explores the effects of different factors on evacuation by real-time updating of the scene or the introduction of predefined probability parameters, such as obstacles, the number and distribution of escape, evacuation instructors and personnel awareness and so on. The optimization mainly focuses on improving the reliability and authenticity of the dynamic model, including the influence of people's vision, export perception on the selection of evacuation routes, the influence of individual differences on the speed of escape and the effect of groups. How to validate the validity of the model becomes the key problem in the study of population aggregation or evacuation model. Because of the characteristics of simulation, validating the effect of model by traditional model based on data analysis and formula calculation can't achieve desired effect. It will be indispensable to build a scene experiment validating the effectiveness of the model. We should not only construct the dynamic model of the evacuation, but also pay more attention to the applicability of the model for pedestrian evacuation and design optimization model. For the applicability of the model, we figure out the source of the model test data in Fig. 4. It can be seen that most articles only use theoretical data testing model, so there is still a lack of implementation of the optimized models.

Proportion of Test Data Sources

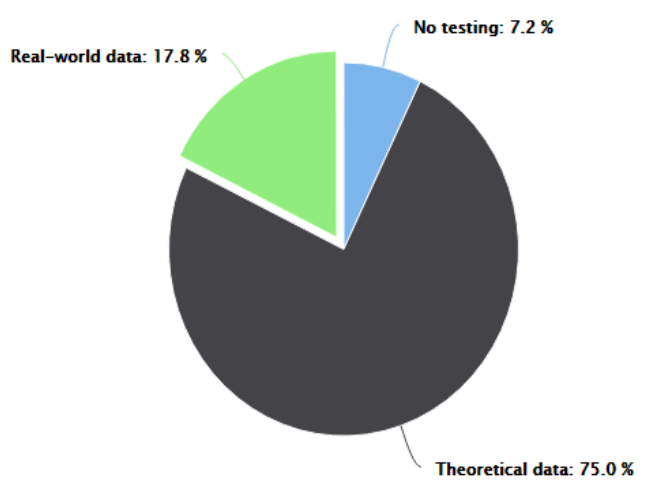

Fig. 4. Percentage of Model Test Data Source

\section{CONCLUSION}

\section{A. System Simulation}

The main research method of emergency evacuation in current study is the system simulation, focusing on building dynamic scenes, simulating the uncertainty of the real scene through the pre-defined probability parameters or real-time updating, bringing more intelligent intelligence and model to enhance the authenticity of interaction between personnel and personnel or personnel and scene. Multi-agent model is widely used in crowd evacuation simulation because of its advantages of autonomy, distribution, coordination, robustness and reliability, as well as the characteristics of multi-level and diversified object-oriented.

\section{B. Applicability of Model}

The study of evacuation in the future population should focus on the optimization of the model and the validation of model, we should not only find the equilibrium point in the reduction of the actual scene uncertainty and the complexity of the model, optimize the model framework to make it close to the reality, but also try to combine the actual data to verify the applicability of the model. Due to the difficulty of actual data collection and the complexity of scene experiment construction, the verification of model will become the hotspot of research.

\section{Direction of Future Research}

The research of emergency evacuation is gradually deepen from the qualitative research to quantitative research. Research on the effects of emergency psychological response on behavioral characteristics based on scene experiments, effects of sensory information, evacuation guidance and trust mechanism on evacuation behavior decision, behavioral characteristics and evacuation patterns of special populations, mixed evacuation model and so on can be used as an important direction for future research.

\section{REFERENCES}

[1] $\mathrm{Xu}$ Min. Investigation on the behavior of different populations in emergency evacuation [A]. Chinese Psychological Society. Proceedings 
of the Eleventh National Conference on psychology 2007:1. Abstract: [C]

[2] Zhan Xin, Yang Lizhong. Study on the SVM neural network model for the selection of evacuation behavior [J]. China Safety Science Journal (CSSJ), 2014, (05): 164-169 (In Chinese)

[3] Graat E, Midden C, Bockholts P. Complex evacuation: Effects of motivation level and slope of stairs on emergency egress time in a sports stadium [J]. Safety Science, 1999, 31(2):127-141.

[4] Tian Yumin. Study on evacuation behavior and evacuation design of special population [J]. Journal of Catastrophology, 2013, 03: 91-94 (In Chinese)

[5] Huo, Feizhou;Song, Weiguo; Chen, Lei; Liu, Chi; Liew, K. M. Experimental study on characteristics of pedestrian evacuation on stairs in a high-rise building[J]. SAFETY SCIENCE, 2016, 07: 165-173

[6] Zhang, Xuefeng; Zhang, Cheng-jun; Bai, Chenxi; Jiang, Taiping; Chu, Yuzhong. Evacuation Model of Multiple Disasters Based on Agent Technology [J]. Journal of System Simulation, 2016, 03: 534-541+568 (In Chinese)

[7] Liu Quanping, Liang Jiahong, Li Meng, Fu Yuewen. Study on Evacuation Behavior Based on Multi - Agent and Cellular Automata [J]. Computer Simulation, 2014, 01: 328-332 (In Chinese)

[8] Dong Chongjie, Liu Yi, Peng Yong. Application of Improved Cuckoo Algorithm in Multi - objective Optimization of Population Evacuation [J]. Journal of System Simulation, 2016, 05: 1063-1069 (In Chinese)
[9] Wang Xia, Xie Zhimin, Guan Xianjun. People in a state of panic evacuation study [C] Micro Simulation International Conference on Future Information Technology and Management Engineering. 2010

[10] Libi Fu, Weiguo Song, Siuming Lo. A fuzzy-theory-based behavioral model for studying pedestrian evacuation from a single-exit room [J]. Physics Letters A, 2016, 06: 165-173

[11] Dewei Li, Baoming Han. Behavioral effect on pedestrian evacuation simulation using cellular automata [J]. SAFETY SCIENCE, 2016, 07: $165-173$

[12] Guo, Xiwei; Chen, Jianqiao; You, Suozhu;.Modeling of pedestrian evacuation under fire emergency based on an extended heterogeneous lattice gas model [J]. PHYSICA A-STATISTICAL MECHANICS AND ITS APPLICATIONS, 2013, 05: 1994-2006

[13] Cao, Shuchao; Song, Weiguo; Lv, Wei. Modeling pedestrian evacuation with guiders based on a multi-grid model [J]. PHYSICS LETTERS A, 2016, 02: 540-547

[14] Xu, Yan; Huang, HaiJun. Simulation of exit choosing in pedestrian evacuation with consideration of the direction visual field [J]. PHYSICA A-STATISTICAL MECHANICS AND ITS APPLICATIONS, 2012, 02.VL 391: 991-1000

[15] Qin Wenhu, Zha Junyuan, Su Guohui, Shu Xin. Research on Simulation Technology of Evacuation Behavior of Population [J]. China Safety Science Journal (CSSJ), 2008, 02: 22-27+177 (In Chinese) 